\title{
Development of a framework for pneumatic device selection for lymphedema treatment
}

\author{
This article was published in the following Dove Press journal: \\ Medical Devices: Evidence and Research \\ 3 December 2009 \\ Number of times this article has been viewed
}

\section{Sonja M Maul' \\ Julie A Devine ${ }^{2}$ \\ Carolyn R Wincer ${ }^{3}$}

'St. Luke's Idaho Elks Rehabilitative Services, Boise, ID, USA; ${ }^{2}$ Center for Wound Care, Butler Memorial Hospital, Butler, PA, USA; ${ }^{3}$ Centers for Rehabilitation Services, Gibsonia, PA, USA
Correspondence: Sonja M Maul 600 North Robbins Road, Suite 10I, Boise, ID 83702, USA

Tel +l 2094894040

Fax + I 2084894064

Email smaul@ierh.org
Abstract: The results of three patients with difficult to manage leg and trunk lymphedema are reported. Patients were treated with the current standard of care: combined decongestive therapy. All three patients met obstacles in achieving independent self-care, which impeded their discharge to home care. The use of two currently available programmable pneumatic compression devices (PCDs) was investigated to determine if these devices could enable these difficult-to-manage patients to meet their discharge goals. Results indicate that programmable PCDs can be effective in managing complicated cases of leg and trunk lymphedema, and that differences may exist between devices. In this trial, the device with lower applied pressures and smaller pneumatic chambers provided better tolerated treatment and overall better patient outcomes. These three case reports show that in-home use of an appropriate PCD can help patients with stage 3 and advanced stage 2 lymphedema achieve stable limb volumes with no exacerbation of lymphedema-related symptoms. Results also demonstrate the ability of patients to consistently use programmable PCDs in a home environment. The results are presented in a structured decision-making framework to aid the therapist and physician in selecting appropriate patients for home PCD lymphedema therapy, and in the selection of an appropriate PCD.

Keywords: lymphedema, swelling, pneumatic, compression, flexitouch, lympha press.

\section{Background and purpose}

Lymphedema is a chronic condition in which excessive fluid and protein accumulate in the interstitial spaces. ${ }^{1}$ The condition occurs from impairment of the lymph system's ability to drain fluids. Lymphedema can be primary in nature (idiopathic), ${ }^{1}$ but (in the US) is most often secondary to acute trauma, surgical intervention, or as a side-effect of cancer treatments. ${ }^{1-5}$

Lymphedema of the skin and subcutaneous tissues is the most common and clinically significant presentation of this condition. ${ }^{2}$ During the early stages, the patient often presents with a feeling of heaviness or tightness of the affected limb, combined with aching or discomfort, impaired mobility, and mild swelling. ${ }^{1,2}$ As swelling increases, connective tissues harden and thicken, and the risk of infection increases. ${ }^{2}$

There is no known cure for lymphedema, and treatments are primarily aimed at minimizing and controlling swelling. The standard treatment for lymphedema is combined decongestive therapy (CDT). ${ }^{3}$ CDT is usually implemented in two phases and aims to halt lymphedema progression and reduce it to a level that can be managed by the patient at home. During phase I, CDT is performed by a therapist in the clinic over multiple intensive therapy sessions that include manual lymph drainage (MLD), gradient compression through the use of short-stretch bandages, decongestive 
exercise, skin care, and wearing of well-fitted compression garments. The length of phase I treatment is variable, but generally lasts from two to four weeks and is concluded when the patient's affected limb(s) reach stable girth and volume measurements. ${ }^{2}$

Once stable girth and volume measurements are achieved, phase II begins and the patient is discharged to follow an at-home self-management regimen. Phase II contains the same elements of phase I, but the patient self-administers MLD, and is responsible for the application and removal of all compression wraps and garments. Successful outcomes depend on the ability of patients to carry out all the elements of phase I at home properly and consistently. Therefore, the role of the lymphedema therapist is not only to treat patients during phase I, but also to educate and train the patient in preparation for phase II. Successful outcomes are only achieved if the day-to-day challenges of self-therapy are anticipated and addressed when devising at-home treatment protocols that meet the reality of everyday life.

Pneumatic compression devices (PCDs) have been available for many years as an alternative or adjunct to MLD (phase I) or self-MLD (phase II). Numerous studies reported positive results and reduced limb volumes with use of PCD treatment. ${ }^{6-19}$ However, most of these treatment protocols were not clinically sustainable, as they were several hours in duration (two to six hours). Additionally, much of the literature is now over a decade old and fails to describe devices with sufficient specificity to provide meaningful treatment guidance with today's newer PCD technologies. Further complicating interpretation of the literature is that some of the older, nonprogrammable PCDs were associated with complications and poor patient acceptance, ${ }^{20,21}$ primarily because older PCDs applied higher static compression than were deemed clinically suitable. ${ }^{22}$ Risks associated with these older, nonprogrammable devices included development of a fibrotic cuff at the limb root, ${ }^{21,23}$ initiation or worsening of genital lymphedema, ${ }^{21}$ and exacerbation of lymphedema in the chest, trunk, and/or abdomen. ${ }^{24}$ Development of truncal lymphedema can lead to growth of fibrosclerotic tissue in those regions, increased pain and disability as well as exacerbation of psychosocial issues, especially when genital lymphedema is present.

The older, nonprogrammable PCDs were designed primarily to treat chronic venous insufficiency and not for lymphedema. These "standard" PCD's are generally noncalibrated, nonprogrammable, nongradient compressors with segmental appliances, and have few chambers and limited adjustability.
Newer PCDs specifically designed for lymphedema are now available. Two such devices utilized in our lymphedema treatment protocols are the Flexitouch ${ }^{\circledR}$ (FT) system (Tactile Systems Technology, Inc., Minneapolis, MN, USA) and the Lympha Press $^{\circledR}$ Optimal $^{\text {TM }}$ (LPO) system (Lympha Press USA, Manalapan, NJ, USA, a subsidiary of Mego Afek AC Ltd, Israel). Both devices are designed with innovative features such as variable interface pressures, therapy sequences, and enhanced programmability designed specifically for lymphedema. These new features address most of the limitations of the older PCD systems.

The FT device uses a mild work and release therapeutic approach designed to simulate MLD. Applied pressures to the skin are variable; averaging 9-13 mm Hg. ${ }^{25}$ Treatment starts proximally in the trunk with a preparatory phase and then proceeds to a drainage phase that works in a distal to proximal sequence. Thirteen available programs provide treatment adjustability. Garment chambers are approximately 1.5-2 inches wide and are designed to treat the adjacent trunk and affected (38.1-50.8 mm) limb. Garments are constructed of a stretchable material that adheres with Velcro ${ }^{\circledR}$ fasteners (Velcro USA Inc., Manchester, NH, USA).

Like the FT system, the LPO device provides a variety of programming options intended to meet a patient's clinical needs including an abdominal pre-therapy phase. Pressure range is identified as between $20-90 \mathrm{~mm} \mathrm{Hg}$ but actual applied pressures have not been reported. The LPO garments have larger 3-5 inch (76.2-127 mm) chambers, and are constructed of a nonstretch nylon with zipper closures. They are available in single and bilateral pant-like and jacket style garments.

Clinicians have used programmable PCDs to enhance patients' ability to manage lymphedema effectively at home. However, the literature is scant with regard to patient and device selection criteria to assist in making specific recommendations as to when such therapy is appropriate, and which device to use. Often these decisions are mandated by third party payer restrictions and not by clinical rationale.

The purpose of this communication is to illustrate, via three case reports, typical challenges presented to the therapist in achieving an appropriate match between patient needs and therapeutic modalities. It will also describe a structured decision-making framework designed to aid the therapist and physician in deciding whether or not PCD therapy may be beneficial and which PCD to recommend.

\section{Case descriptions}

Three case reports are presented for individuals experiencing significant challenges with effective phase I lymphedema 
management that precluded successful advancement to phase II treatment. In an effort to address individual barriers and find solutions, home PCD treatment was initiated for each patient. Trials of two programmable PCDs were conducted, and outcomes were assessed by the treating lymphedema therapist.

This study was conducted in compliance with Good Clinical Practice (US Food and Drug Administration), ${ }^{26}$ and the Health Insurance Portability and Accountability Act regulations (US Department of Health and Human Services). ${ }^{27}$ All patients gave consent for use and publication of their clinical data.

\section{Case I}

\section{Patient history}

The patient was a 76-year-old female diagnosed (at age 70 years) with primary lipolymphedema of the bilateral lower extremities and trunk. Her right leg measured larger than her left, with moderate involvement of her feet. She had significant history of tenderness in her legs, swelling, insomnia, recurrent cellulitis infections, bruising, chronic venous insufficiency, and rheumatoid arthritis.

\section{Examination}

On June 15, 2008, she presented with bilateral lower extremity, stage 3 lipolymphedema with truncal and feet involvement, tenderness upon palpation of the tissues, a positive Stemmer's sign, and deepening skin folds at the ankles. Functionally, she demonstrated reduced ability to bend at the waist, knees, and ankles and experienced muscle fatigue with mild activity or exercise. She reported daily pain level at six on a $0-10$ numeric pain scale $(6 / 10)$, zero meaning no pain and 10 meaning the worst pain possible, with a burning sensation at night and poor tolerance for pressure on her skin or abdomen.

\section{Intervention}

The patient was seen in-clinic for CDT every other workday. One leg was treated at a time due to restricted mobility and nausea upon exertion. The initial focus was on the right leg. From the onset, she could not tolerate the thigh bandages; consequently, the therapist only bandaged the lower leg. The patient could not maintain the prescribed self-MLD, as the effort often caused nausea with headaches.

After four weeks of CDT, her right leg volume reduction (18\%) plateaued (Figure 1), but significant issues remained. She had not achieved targeted volume reduction, and still exhibited significant edema marked by a cuff above the ankle. Treatment was then initiated on the left leg, and target volume reductions $(7.6 \%)$ were quickly achieved. Within three weeks, left leg volume reduction had reached a plateau. Despite the positive gains on the left leg, the right leg experienced volume increases when bandages were removed and/or when therapy visits were more than three days apart.

At the conclusion of her 10-week in-clinic CDT, the therapist was challenged to define a home program that the patient could successfully implement. The therapist conducted an

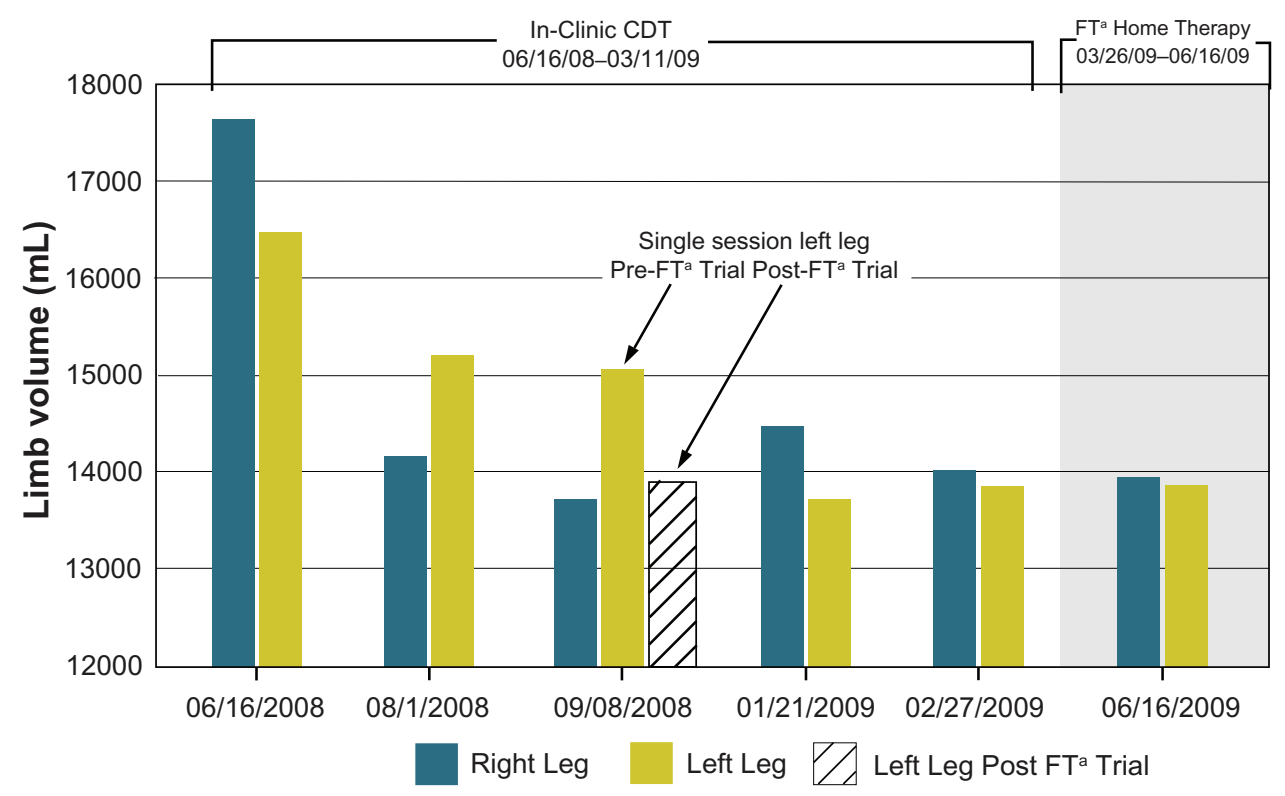

Figure I Case report I: bilateral leg volume changes. Note: ${ }^{\mathrm{F} T}$, flexitouch. 
in-clinic trial of the FT device on the left side only. The leg and trunk garments were applied, and the device was programmed for the one-hour lower extremity treatment. The patient responded well to the treatment stating it felt relaxing, and left limb volume decreased by $7.8 \%$ post-treatment.

Prior to approving payment, the patient's health insurer required a one-month trial of the LPO device, as it was available within their network. An in-clinic trial with the LPO device was conducted by the therapist and LPO representative. The LPO was programmed to its lowest available setting (31 mm Hg displayed setting) using the pre-therapy mode (starting at the thigh) in preparation for the one-hour sequence on both legs. The therapist omitted use of the truncal treatment due to the patient's history of poor tolerance and nausea with abdominal pressure. Twenty-five minutes into the hour-long treatment, the patient reported nausea and heart palpitation. The therapist immediately discontinued the trial and removed the garment. The patient's blood pressure was elevated to $156 / 86$, above her normal pressure of 130/70.

\section{Outcome and follow-up}

Based on the trial outcomes with both PCDs the patient's insurer authorized payment for the FT device. At her threemonth follow-up visit, she exhibited sustained limb volume reductions, continued tolerance of the FT device with minimal pain, improved sleep, no infections, lessened report of fatigue during activity, and ease of bending at the waist for donning her below knee FarrowWrap ${ }^{\mathrm{TM}}$ garment (Farrow Medical Innovations, Bryan, TX, USA).

\section{Case 2}

\section{Patient history}

The patient was a 57 -year-old male with stage 3 primary lymphedema of both legs due to Milroy's disease. He had multiple hospitalizations for wounds and recurrent cellulitis infections, penicillin resistance, high blood pressure, and congestive heart failure controlled with stent placement. His lymphedema had progressed into his trunk, back, and genitals, and he had difficulty wearing shoes. His lymphedema impaired his ability to participate in normal activities such as walking, climbing stairs, carrying heavy loads, bending, kneeling, or standing. His work week often required 60 hours of being on his feet, but this was becoming difficult due to advanced swelling in his legs and abdomen.

\section{Examination}

The lymphedema therapist documented the patient's functional impairments, including poorly controlled swelling in his abdomen, genitals, legs, and feet, as well as continuous (5/10) leg and foot pain, moderate to severe fibrotic skin changes in the lower extremities, chronic mycosis, and positive Stemmer's sign bilaterally. The patient could not reach his feet because abdominal edema limited his ability to bend forward. He could not adequately lift his legs to reach his feet because his leg muscles were weak. His gait was awkward, and he had an impaired body image. The therapist recommended in-clinic therapy five times weekly for eight weeks, but the patient was only able to attend therapy three times weekly due to his work schedule.

\section{Intervention}

The patient began in-clinic CDT three times a week which continued for approximately two months. The therapist treated one leg at a time to avoid excessive fluid loads returning to the central circulation. The right leg was treated during the first month followed by the left leg during the second month.

Significant limitations emerged for self care during phase I therapy. The patient resisted the therapist's recommendation for complete leg to waist compression during the day because it impaired his ability to work. He was also unable to effectively complete self-MLD due to fatigue and limited endurance. His leg muscles were weak, and he was unable to hold positions for bandaging, self-MLD, or adequate completion of decongestive exercise. Consequently, the therapist suggested implementing adjunctive treatment using a programmable PCD with truncal treatment.

An in-clinic trial of the FT device was conducted on the right leg and the hour-long treatment was well tolerated. The therapist calculated a $2.8 \%$ volume reduction in the right leg after the first treatment (Figure 2) and documented a softening of the fibrosis in his foot and leg. He was able to use the device with minimal assistance, and indicated willingness to continue use at home because it relieved his leg pain.

After several months of review, the patient's insurance company determined that a programmable PCD was medically necessary for the patient, but indicated it would only pay for the LPO device because it was available within their network. The therapist arranged a trial of the LPO device and the system was set to its lowest setting for the preparatory program. During the one-hour treatment, the patient reported pain "like having a blood pressure cuff on my legs," and indicated that he would not be able to sustain the treatment at home. At the completion of the in-clinic trial, the therapist gauged no change in the hardness of the fibrosis, and no further measurements were taken. Insurance coverage was sought and obtained for the FT device. 


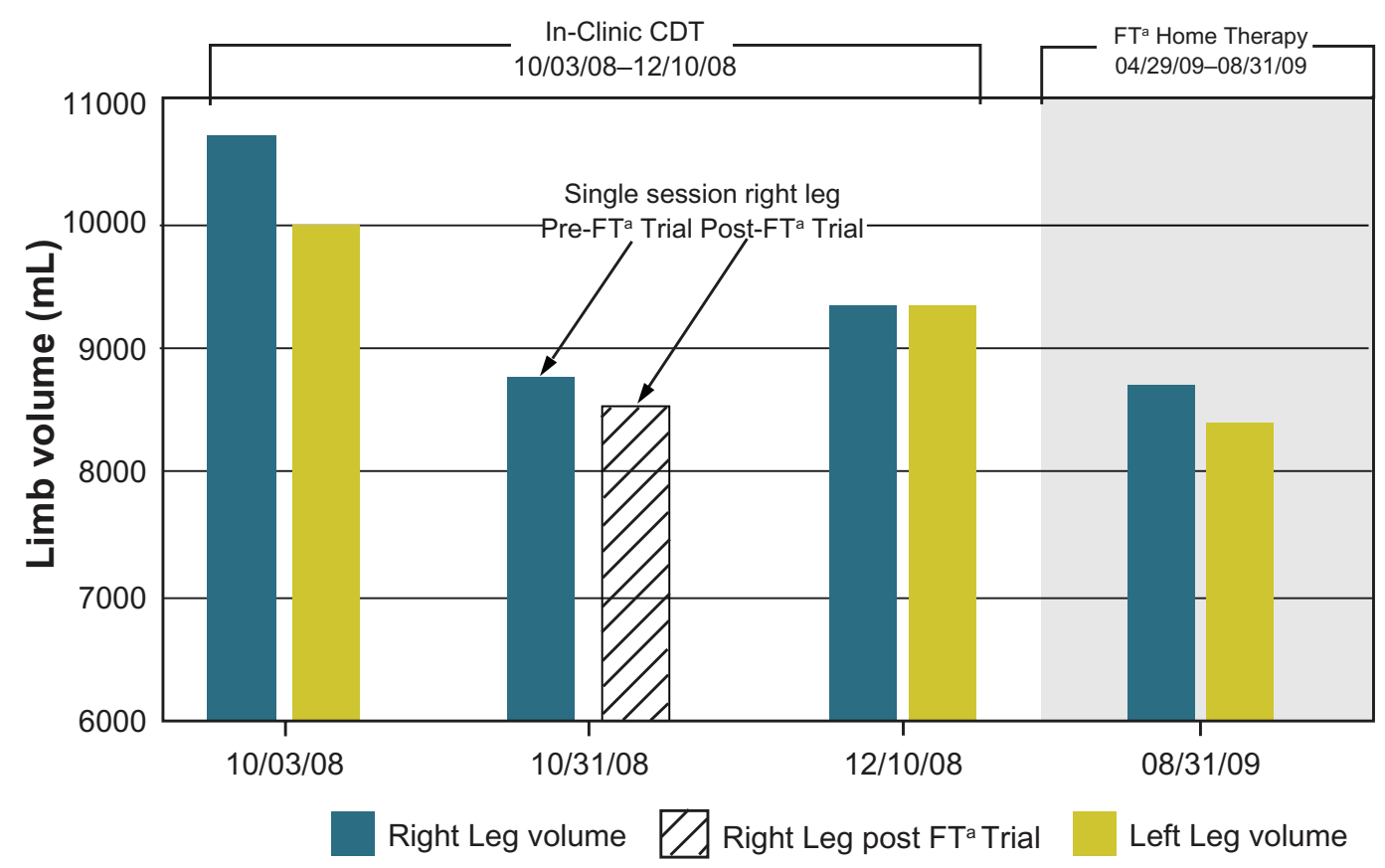

Figure 2 Case report 2: bilateral leg volume changes.

Note: ${ }^{\mathrm{a} T T}$, flexitouch.

\section{Outcome and follow-up}

Approximately four months after initiating daily home PCD therapy with the FT device, the patient experienced sustained limb volume reductions and no lymphedema-related complications. He was able to manage the lymphedema on his own, had no exacerbation of genital lymphedema, reported less leg fatigue while standing, walking, climbing stairs, carrying heavy loads, bending at the waist, and continued to work the hours required for his job with no recurrence of infections or wounds.

\section{Case 3}

\section{Patient history}

The patient was a 63-year-old female with stage 2 lymphedema of her left leg and adjacent trunk secondary to excision of her left inguinal lymph nodes due to metastasized Merkel cell skin cancer and subsequent radiation therapy. She also had rheumatoid arthritis and diabetes mellitus.

\section{Examination}

Initial left leg limb volume was $16.8 \%$ greater than her right leg (Figure 3) with fibrosis present in her left pelvis, hip, and leg. The lymphedema therapist noted that her left leg was stiff and inflexible and that her left foot was fibrotic and reddened. She also exhibited compromised skin integrity at the left pelvis, poor pressure tolerance on her skin nearest the pelvic scar, and impaired left hip range of motion ( $90^{\circ}$ flexion, $10^{\circ}$ extension). The patient indicated increased fatigue since her surgery and expressed concern about her changing body image. She also reported left leg pain of $9 / 10$, worsening as the day progressed. At home she did very little because she was fatigued, and her sleep was often interrupted by leg pain. She reported that bending at the hip is nearly impossible, that she could not dangle her leg, and that she climbed stairs one at a time due to limited range of motion (ROM). She also reported difficulty in getting in and out of chairs, bed, and automobiles.

\section{Intervention}

Twice-weekly in-clinic CDT was initiated and continued for six weeks. However, the patient voiced frustration because of the discomfort and feelings of claustrophobia associated with constantly wearing bandages. Additionally, her leg volume had increased. The therapist assessed that the patient would benefit from PCD treatment on her leg at home.

The therapist arranged an in-clinic trial of the FT device. Pre and post measures were not recorded. The patient was able to use the device with minimal assistance and indicated that the treatment was very relaxing. Request for the FT device was denied by the patient's health insurance plan because the LPO device was available within their network. The denial letter indicated that if the LPO device was assessed as not clinically appropriate after a one-month trial, that the FT system would be reconsidered with appropriate 


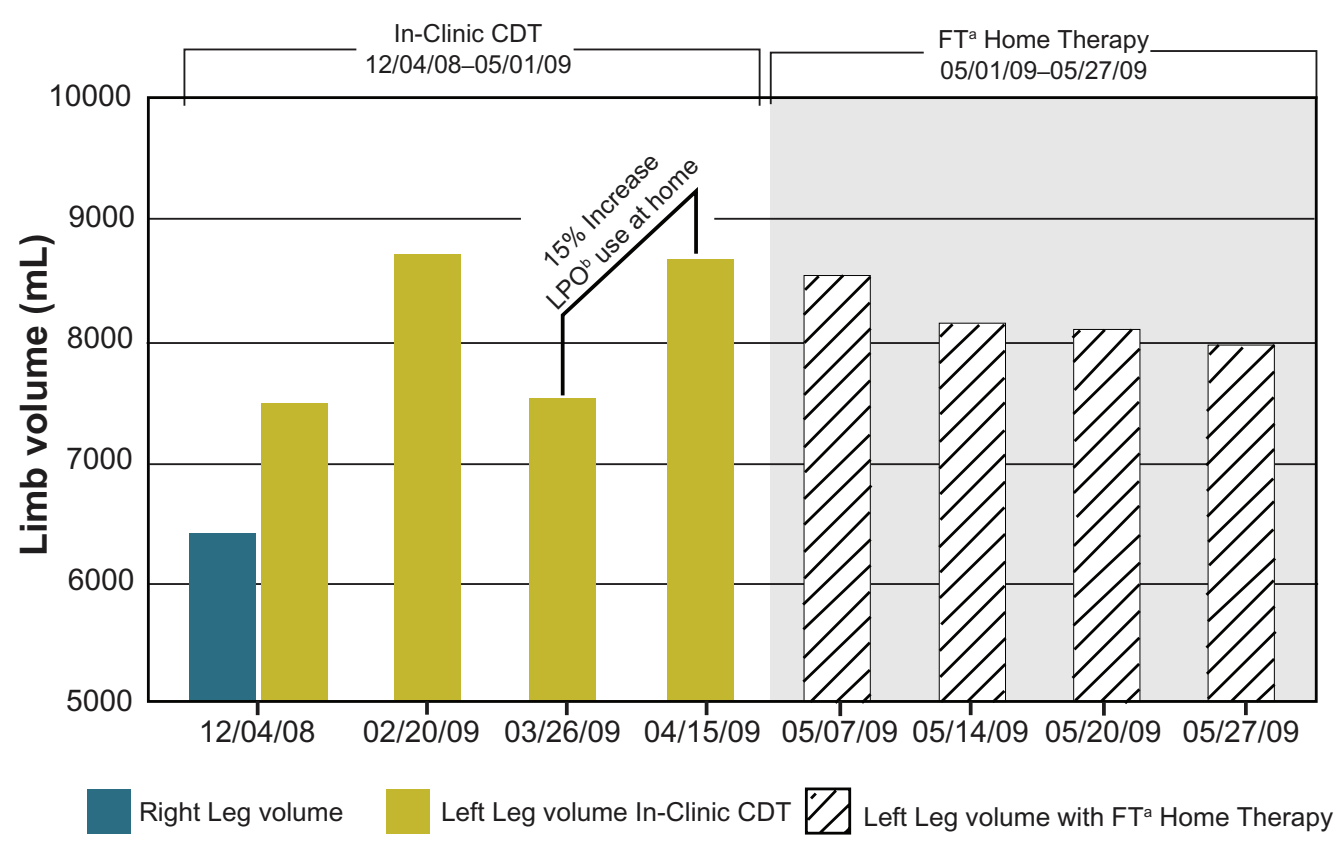

Figure 3 Case report 3: left leg volume changes. Notes: ${ }^{\mathrm{a} T T}$, flexitouch; ${ }^{\text {L }} \mathrm{PO}$, lympha press optimal.

documentation. An in-clinic trial of the LPO device was conducted. The patient required minimal assistance in using the device; however, she asked to stop midway through the treatment because it was uncomfortable and made her nauseous. She continued attempts to use the device at home, hoping for increased tolerance over time.

After three weeks of home use of the LPO device, left leg volume had increased by $15 \%$ and there was substantial increase in fibrosis. The clinical notes described the patient as having a severely antalgic gait pattern on the left lower extremity, and the patient reported an 8/10 pain level to left buttock. The therapist recommend discontinuation of the LPO device due to increased edema, pain, and lack of tolerance to treatment. In-clinic MLD was continued in combination with a compression garment. The therapist pursued appeal for the FT device with the patient's health insurer, and authorization was granted.

\section{Outcome and follow-up}

Twice weekly clinic visits and daily home use of the FT device showed decreasing left leg volumes, reduced pain levels to 3-4/10, and softening fibrosis in her foot and toes. After approximately one month of treatment, left leg edema volumes were reducing, and there was further fibrosis softening, pain levels reduced to $1 / 10$, left hip flexion was $100^{\circ}$ and extension $20^{\circ}$ and her gait pattern improved. The patient also reported that she could wear compression stockings comfortably up to 12 hours a day and be on her feet at work.

\section{Discussion}

Self-care limitations and complications which emerge during phase I of CDT therapy need to be assessed before a patient is discharged to at home phase II therapy. There are significant risks associated with a patient's inability to carry out the various elements of phase II, including a loss of gains achieved during phase I and worsening of the lymphedema with subsequent development of lymphedema-related complications. These complications can range from decreased mobility and ROM to serious recurrent infections. In such cases, loss of independence, deterioration in quality of life, impaired body image, and increased frequency of in-clinic therapy and associated costs become continuing burdens for the patient. ${ }^{1,3,28}$ Thus, it is crucial that clinicians identify patient limitations and derive suitable therapies during phase I. To realize this, therapists need options for their patients to progress to the discharge plan, and patients need effective treatment that they can implement at home.

In these case reports, three individuals presented their therapists with challenges and functional limitations that hampered control of their lymphedema during phase I of treatment. Consequently, the likelihood of long-term phase II success was limited, necessitating alternate means 
of assisting the patient in achieving an acceptable outcome. Incorporating use of a programmable PCD is one strategy clinicians may consider to support successful management of lymphedema at home. ${ }^{29}$ However, there is scant literature to guide clinicians in the appropriate selection and use of a PCD device, ${ }^{4}$ and established professional organizations do not provide specific recommendation or standards for device selection. ${ }^{30,31}$ Manufacturer literature offers some guidance, but this can be biased and is specific to the manufacturer's device.

In each of the three cases, the clinician determined that adjunctive use of a programmable PCD may benefit the patient, and two possible products were identified for in-clinic trial. Each device provided appliances that treated not only the affected leg but also the trunk. Each device also provided a wide range of features and adjustability to address the specific clinical condition of lymphedema. Three significant differences existed between the two PCD's:

1. The LPO device utilized a garment that treated bilaterally at the same time. While some may view this as a convenient means to reduce treatment time, therapists have raised concerns about the possibility of causing fluid overload to the trunk.

2. The mechanism of applied pressure appeared to be different between the two devices. The FT device provided brief applications of lower pressure through smaller garment chambers than the LPO device. Our observation was that with the FT device, no two chambers were inflated at the same time during the course of treatment. In addition to the LPO device chambers being larger, we observed the application of pressure in a more static inflate and hold treatment pattern, with multiple chambers remaining inflated simultaneously.

3. The applied interface treatment pressure appears to be lower for the FT device based on patient experience and the reported mechanism of action (average 9-13 mm Hg) ${ }^{25}$ than for the LPO device $(31 \mathrm{~mm} \mathrm{Hg}$ displayed pressure).

In all three cases, in-clinic trials of the two programmable PCDs provided compelling outcomes from which the clinicians were able to assess the device most suitable for the patient. These cases illustrate the importance of such trials as a means of assessing potential phase II problems with device use. Figures 4 and 5 illustrate essential elements of these processes via diagrams to aid in decision making. Although not inclusive of all circumstances, these flow diagrams provide a framework as guidance for when home PCD use might benefit patients.

These three cases demonstrate that programmable PCDs may provide effective adjunctive therapy to help manage patients with difficult to treat lymphedema. Outcomes obtained in these cases stress the importance of conducting in-clinic trials to best match the available devices to the patient. In these three cases, third party payer network contracts mandated use of a particular device, while in-clinic trials and clinician preference indicated an alternative and more appropriate choice. In such cases, the clinician must be willing to work with the patient and insurer in obtaining payment for the device that is most clinically appropriate for the individual patient.

The outcomes presented here cannot be generalized because of the small number of cases and the potential
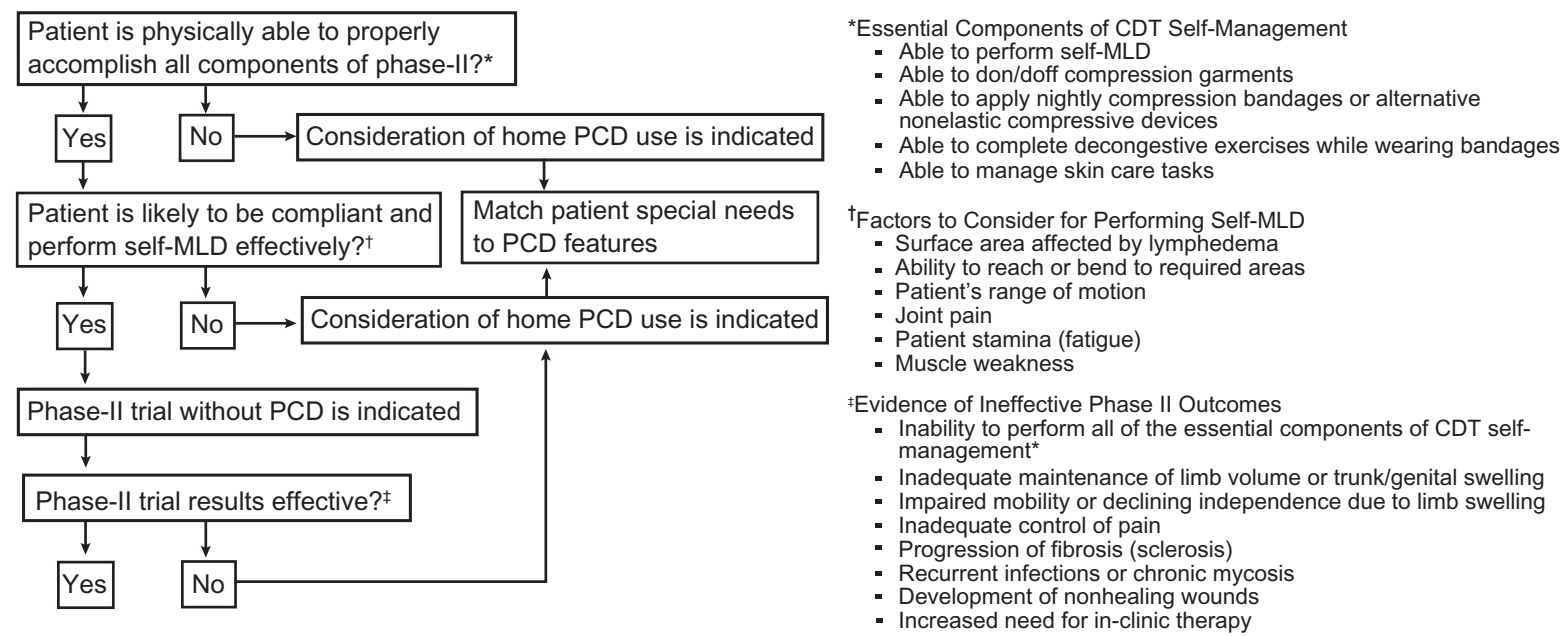

Figure 4 This diagram indicates the essential elements to be considered when deciding if use of a PCD should be considered. Abbreviations: CDT, combined decongestive therapy; MLD, manual lymph drainage; PCD, pneumatic compression device. 


\section{Decision tree for home PCD selection}

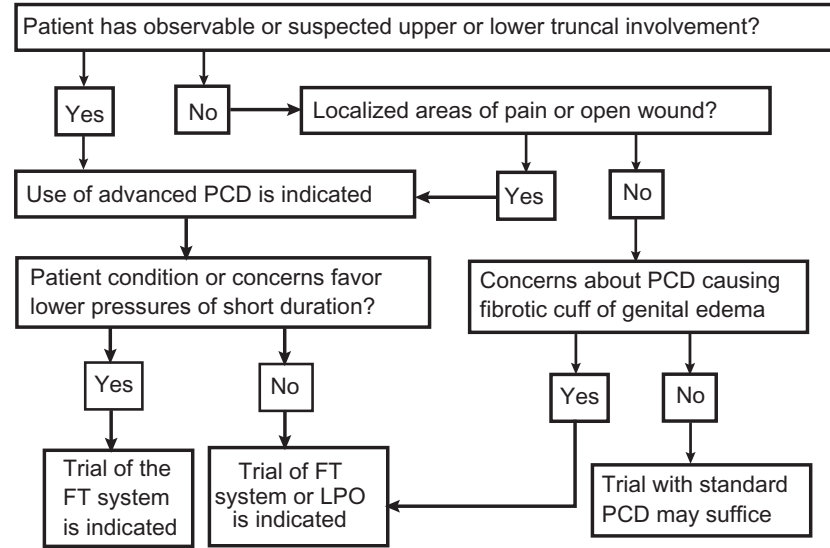

Figure 5 This diagram indicates the essential elements to be considered when deciding which PCD to recommend once the decision is made that a PCD is indicated. Abbreviation: LPO, Lympha Press ${ }^{\circledR}$ Optimal ${ }^{\mathrm{TM}}$; FT, Flexitouch ${ }^{\circledR}$; PCD, pneumatic compression device.

for clinician bias. However, the resulting device selection framework should serve as a useful tool for lymphedema practitioners wanting to incorporate programmable PCD therapy into their practice. Larger scale, prospective, randomized clinical trials designed to address patient selection criteria would be appropriate to further assess and refine this framework. The authors hope this is the beginning of additional clinical research to investigate outcomes and treatment guidelines for home PCD use.

\section{Acknowledgments}

The authors wish to thank Mr James B Lutz, MS, CCRA, Lutz Consulting LLC, who provided medical writing services on behalf of Tactile Systems Technology, Inc. The authors report no conflicts of interest in this work.

\section{References}

1. Ridner SH, McMahon E, Dietrich MS, Hoy S. Home-based lymphedema treatment in patients with cancer-related lymphedema or noncancerrelated lymphedema. Oncol Nurs Forum. 2008;35(4):671-680.

2. Lawenda BD, Mondry TE, Johnstone PA. Lymphedema: a primer on the identification and management of a chronic condition in oncologic treatment. CA Cancer J Clin. 2009;59(1):8-24.

3. Wilburn O, Wilburn P, Rockson SG. A pilot, prospective evaluation of a novel alternative for maintenance therapy of breast cancer-associated lymphedema [ISRCTN76522412]. BMC Cancer. 2006;6:84.

4. Cheville AL, McGarvey CL, Petrek JA, Russo SA, Taylor ME, Thiadens SR. Lymphedema management. Semin Radiat Oncol. 2003;13(3):290-301.

5. Donachy JE, Christian EL. Physical therapy intervention following surgical treatment of carpal tunnel syndrome in an individual with a history of postmastectomy lymphedema. Phys Ther. 2002;82(10):1009-1016.

6. Raines JK, O'Donnell TF Jr. Kalisher L, Darling RC. Selection of patients with lymphedema for compression therapy. Am J Surg. 1977;133(4):430-437.
7. Swedborg I. Effects of treatment with an elastic sleeve and intermittent pneumatic compression in post-mastectomy patients with lymphoedema of the arm. Scand J Rehabil Med. 1984;16(1):35-41.

8. Zanolla R, Monzeglio C, Balzarini A, Martino G. Evaluation of the results of three different methods of postmastectomy lymphedema treatment. J Surg Oncol. 1984;26(3):210-213.

9. Richmand DM, O'Donnell TF Jr, Zelikovski A. Sequential pneumatic compression for lymphedema. A controlled trial. Arch Surg. 1985;120(10):1116-1119.

10. Zelikovski A, Bair A, Haddad M, Reiss R. The mobile pneumatic arm sleeve: a new device for treatment of arm lymphedema. Lymphology. 1985;18(2):68-71.

11. Kim-Sing C, Basco VE. Postmastectomy lymphedema treated with the Wright linear pump. Can J Surg. 1987;30(5):368-370.

12. Klein MJ, Alexander MA, Wright JM, Redmond CK, LeGasse AA. Treatment of adult lower extremity lymphedema with the Wright linear pump: statistical analysis of a clinical trial. Arch Phys Med Rehabil. 1988;69(3 Pt 1):202-206.

13. Pappas CJ, O'Donnell TF, Jr. Long-term results of compression treatment for lymphedema. J Vasc Surg. 1992;16(4):555-562; discussion 562-564.

14. Bunce IH, Mirolo BR, Hennessy JM, Ward LC, Jones LC. Postmastectomy lymphoedema treatment and measurement. Med J Aust. 1994;161(2):125-128.

15. Johansson K, Lie E, Ekdahl C, Lindfeldt J. A randomized study comparing manual lymph drainage with sequential pneumatic compression for treatment of postoperative arm lymphedema. Lymphology. 1998;31(2):56-64.

16. Dini D, Del Mastro L, Gozza A, et al. The role of pneumatic compression in the treatment of postmastectomy lymphedema. A randomized phase III study. Ann Oncol. 1998;9(2):187-190.

17. Miranda F Jr, Perez MC, Castiglioni ML, et al. Effect of sequential intermittent pneumatic compression on both leg lymphedema volume and on lymph transport as semi-quantitatively evaluated by lymphoscintigraphy. Lymphology. 2001;34(3):135-141.

18. Petrek JA, Pressman PI, Smith RA. Lymphedema: current issues in research and management. CA Cancer J Clin. 2000;50(5):292-307; quiz 308-311.

19. Szuba A, Achalu R, Rockson SG. Decongestive lymphatic therapy for patients with breast carcinoma-associated lymphedema. A randomized, prospective study of a role for adjunctive intermittent pneumatic compression. Cancer. 2002;95(11):2260-2267.

20. Lynnworth L. Pump survey of members of the greater Boston lymphedema support group. 1997. Available from: http://www.gbln. org/survey.htm. Accessed on November 10, 2009.

21. Boris M, Weindorf S, Lasinski BB. The risk of genital edema after external pump compression for lower limb lymphedema. Lymphology. 1998;31(1):15-20.

22. Segers P, Belgrado JP, Leduc A, Leduc O, Verdonck P. Excessive pressure in multichambered cuffs used for sequential compression therapy. Phys Ther. 2002;82(10):1000-1008.

23. Casley-Smith J, Casley-Smith JR. Other treatments for lymphoedema. 1998. Available from: http://www.lymphoedema.org.au/tret_oth.html. Accessed on November 10, 2009.

24. Földi M, Földi E, Kubik S. Textbook of Lymphology: For physicians and lymphedema therapists. München, Germany: Urban and Fischer; 2003.

25. Mayrovitz HN. Interface pressures produced by two different types of lymphedema therapy devices. Phys Ther. 2007;87(10):1379-1388.

26. US Food and Drug Administration. Good Clinical Practice. 21 C.F.R. Parts 11, 40, 54, 56, 312, 314. Available from: http://www.fda.gov/oc/ gcp/regulations.html. Accessed on May 13, 2009.

27. US Department of Health and Human Services. Standards for Privacy of Individually Identifiable Health Information; Final Rule, 45 C.F.R. Parts 160, and 164. Code of Federal Regulations Available from: http://wedi.org/ snip/public/articles/45CFR160\&164.pdf. Accessed on May 13, 2009.

28. Hammond T. Reduction of complications and associated costs with flexitouch $^{\circledR}$ therapy for lymphedema. The Open Rehabilitation Journal. $2009 ; 2: 54-57$. 
29. Cannon S. Pneumatic compression devices for in-home management of lymphedema: two case reports. Cases Journal. 2009;2:6625.

30. International Society of Lymphology. Position statement of the National Lymphedema Network. 2006. Available from: http:/www.lymphnet. org/pdfDocs/nlntreatment.pdf. Accessed on November 10, 2009.
31. International Society of Lymphology. The diagnosis and treatment of peripheral lymphedema: 2009 consensus document. Lymphology. 2009;42:51-60.

\section{Publish your work in this journal}

Medical Devices: Evidence and Research is an international, peerreviewed, open access journal that focuses on the evidence, technology, research, and expert opinion supporting the use and application of medical devices in the diagnosis, treatment and management of clinica conditions and physiological processes. The identification of novel devices and optimal use of existing devices which will lead to improved clinical outcomes and more effective patient management and safety is a key feature. The manuscript management system is completely online and includes a quick and fair peer-review system. Visit http://www. dovepress.com/testimonials.php to read real quotes from authors.

Submit your manuscript here: http://www.dovepress.com/medical-devices-evidence-and-research-journal 